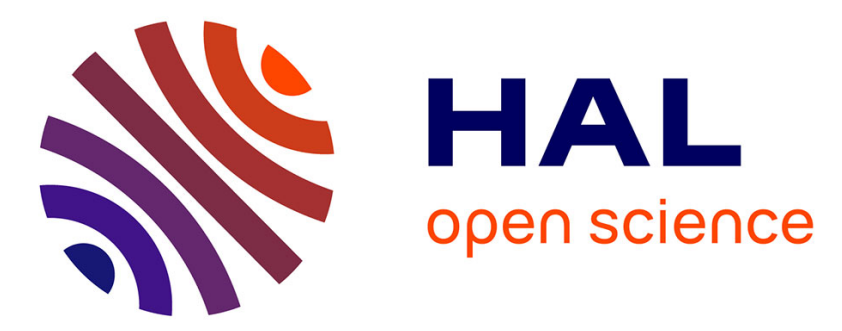

\title{
Effets d'auto-absorption avec une source Mössbauer de 57 Co dans du fer, isotrope ou polarisée linéairement
}

\author{
M. Henry, F. Varret
}

\section{To cite this version:}

M. Henry, F. Varret. Effets d'auto-absorption avec une source Mössbauer de 57Co dans du fer, isotrope ou polarisée linéairement. Revue de Physique Appliquée, 1979, 14 (1), pp.289-294. 10.1051/rphysap:01979001401028900 . jpa-00244588

\section{HAL Id: jpa-00244588 https://hal.science/jpa-00244588}

Submitted on 1 Jan 1979

HAL is a multi-disciplinary open access archive for the deposit and dissemination of scientific research documents, whether they are published or not. The documents may come from teaching and research institutions in France or abroad, or from public or private research centers.
L'archive ouverte pluridisciplinaire HAL, est destinée au dépôt et à la diffusion de documents scientifiques de niveau recherche, publiés ou non, émanant des établissements d'enseignement et de recherche français ou étrangers, des laboratoires publics ou privés. 


\title{
Effets d'auto-absorption avec une source Mössbauer de ${ }^{57}$ Co dans du fer, isotrope ou polarisée linéairement
}

\author{
M. Henry et F. Varret \\ Groupe de Physique et Chimie du Solide $\left({ }^{*}\right)$, Faculté des Sciences du Mans
} F 72017 Le Mans Cedex, France

(Reçu le 24 mai 1978, révisé le 19 septembre 1978, accepté le 29 septembre 1978)

\begin{abstract}
Résumé. - Nous donnons une expression simple de l'intensité des raies Mössbauer, tenant compte de l'effet d'auto-absorption. Cette expression permet de rendre compte des intensités mesurées avec une source de ${ }^{57} \mathrm{Co}$ dans du fer, en l'absence et en présence d'un champ magnétique appliqué $(0,4 \mathrm{~T})$. On en déduit, entre autres résultats, l'épaisseur effective de la couche traversée par le rayonnement, $3,5 \pm 1$.
\end{abstract}

Abstract. - We calculate the intensity of a Mössbauer line submitted to self-absorption effects. A simple formula is derived, which is used to interpret the measured intensities of a ${ }^{57} \mathrm{Co}$ on Fe source in two cases : zero external field ; $0.4 \mathrm{~T}$ applied field. The effective thickness of the source is determined : $3.5 \pm 1$.

1. Introduction. - Les sources Mössbauer dans des matrices magnétiques (par exemple ${ }^{57} \mathrm{Co}$ dans $\mathrm{Fe}$ ) servent habituellement à des expériences de polarisation [1]. Souvent il faut mesurer avec précision l'intensité des raies du spectre obtenu, et l'effet d'autoabsorption sur l'intensité des raies d'émission de la source n'est pas a priori négligeable, en particulier si la matrice est faite du même élément que l'isotope Mössbauer. Nous avons donc entrepris, comme préliminaire à des expériences de polarisation, de mettre en évidence ces effets d'auto-absorption.

La possibilité de comparer la source dans son état d'orientation isotrope (sans champ appliqué), et dans un état de polarisation rectiligne quasi totale (champ magnétique appliqué perpendiculairement à la direction d'observation) permet $a$ priori d'observer des effets d'auto-absorption d'importance différente, donc de recouper les résultats.

Par ailleurs, l'article récent de Blamey [2] concernant l'intensité de raies d'absorption, nous a donné l'espoir de mettre sous forme simple les expressions décrivant l'auto-absorption.

2. Résultats expérimentaux. - La source utilisée est de fabrication standard (Amersham, ref. CTD 14, activité $10 \mathrm{mCi}$ ). Le ${ }^{57} \mathrm{Co}$ est diffusé dans une couche de fer métallique déposée sur support de cuivre. Ce dépôt de fer a un aspect granuleux; il conduit à des intensités de raies beaucoup plus voisines de celles

(*) E.R.A. C.N.R.S. no 682 . d'une poudre de fer que de celles d'une lamelle, et l'on peut, en première analyse, considérer ce dépôt comme une poudre.

Le dispositif expérimental est représenté sur la figure 1 .

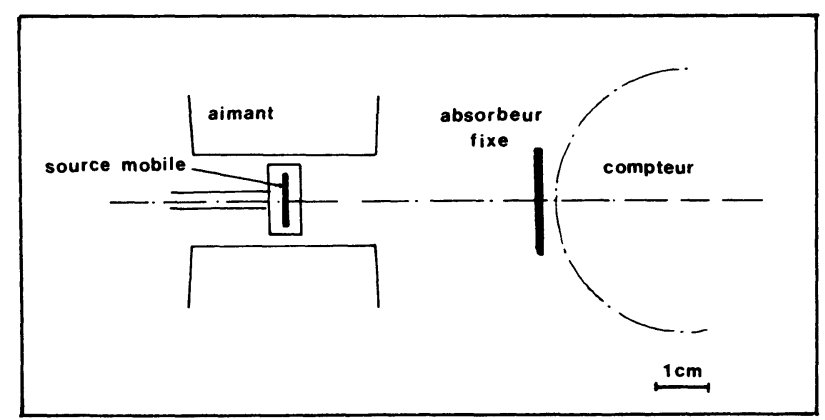

Fig. 1. - Dispositif expérimental de caractéristiques géométriques suivantes : diamètre de l'absorbeur $20 \mathrm{~mm}$; distance source absorbeur $50 \mathrm{~mm}$, entrefer de l'aimant $17 \mathrm{~mm}$.

[Schematic illustration of our system : absorber's diameter $=20 \mathrm{~mm}$, distance between source and absorber $=50 \mathrm{~mm}$, air gap of the magnet $=17 \mathrm{~mm}$.]

Les autres caractéristiques de l'expérience sont les suivantes :

- spectromètre fonctionnant en mode triangulaire symétrique, ce qui élimine, par repli du spectre, la courbure du fond continu,

- champ magnétique de 0,4 tesla fourni par un aimant permanent (champ perpendiculaire à la direction de propagation du rayonnement), 
- absorbeurs d'acier inoxydable (fourni par Amersham, épaisseur $0,44 \mathrm{mg}{ }^{57} \mathrm{Fe} / \mathrm{cm}^{2}$ ) et de ferrocyanure de sodium $\left(0,21 \mathrm{mg}{ }^{57} \mathrm{Fe} / \mathrm{cm}^{2}\right)$.

Les spectres obtenus, indiqués sur la figure 2 , ont conduit aux résultats rassemblés dans le tableau I. Les intensités mesurées sont proportionnelles aux surfaces des raies, supposées Lorentziennes; elles révèlent un écart par rapport aux valeurs idéales, calculées sans auto-absorption. L'interprétation de cet écart fait l'objet du présent travail.

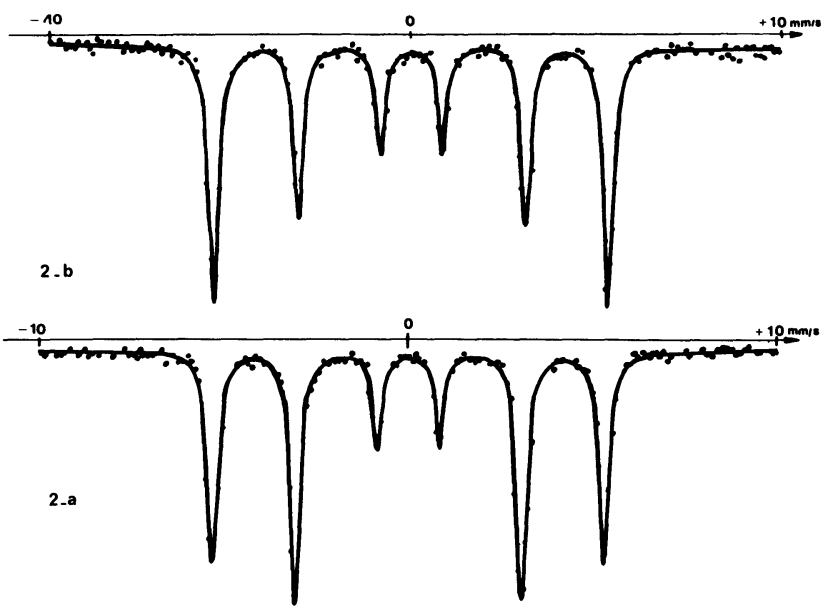

Fig. 2. - Spectres expérimentaux (en trait plein : spectre ajusté) : a) avec champ appliqué ; $b$ ) en champ nul. (On ne présente pas les spectres obtenus avec l'absorbeur d'inox, qui sont très semblables.)

[Experimental spectra (full line : computer-fitted spectra) $: a$ ) with external magnetic field, $b$ ) without external magnetic field (with a stainless steel absorber the experimental spectra are similar).]

3. Effet d'auto-absorption sur une raie Mössbauer. Pour décrire les effets d'auto-absorption utilisons une expression analogue à celle de Margulies et Ehrman [3].

$$
\begin{aligned}
P_{i}= & P_{0} \frac{\Gamma}{2 \pi} \int_{-\infty}^{+\infty} \mathrm{d} E \int_{0}^{+\infty} \frac{C_{i}}{E^{2}+\Gamma^{2} / 4} \rho(x) \times \\
& \times \exp \left\{- \text { fnao }_{0} \frac{C_{i}^{\prime} \Gamma^{2} / 4}{E^{2}+\Gamma^{2} / 4} x\right\} \mathrm{d} x
\end{aligned}
$$

où $P_{0}=$ intensité résonnante initiale,

$P_{i}=$ intensité émergente de la raie $i$ (après autoabsorption),

$f=$ probabilité d'absorption résonnante,

$n a=$ nombre d'atomes Mössbauer par unité de volume,

$C_{i}, C_{i}^{\prime}=$ probabilité relative d'émission (ou d'absorption) pour la raie $i$,

$\rho(x)=$ distribution des atomes émetteurs le long de l'axe $x$ de propagation du rayonnement,

$\Gamma=$ largeur de raie à mi-profondeur.

L'équation (1) est donnée pour le cas d'une source monochromatique; dans le cas d'une source à raies multiples elle s'applique encore si les raies sont bien résolues : c'est notre hypothèse ici. (Il faudrait, théoriquement, tenir compte des effets de réémission, tels que la biréfringence et la rotation Faraday [1, 9] ; ces effets apparaîtraient au paragraphe 5. En pratique, ils sont négligeables parce que l'épaisseur effective mise en jeu dans nos expériences est beaucoup plus faible que celle des absorbeurs utilisés dans les expériences citées $[1,9]$.)

Considérons que la source présente une distribution uniforme de noyaux émetteurs sur son épaisseur $t$ :

$\rho(x)=\left\{\begin{array}{lr}N \text { atomes/volume unité } & 0 \leqslant x \leqslant t \\ 0 & x>t .\end{array}\right.$

L'équation (1) se transforme, selon les mêmes auteurs [3], pour donner :

$$
\begin{aligned}
\frac{P_{i}}{P_{0}}= & \frac{N t}{T} \frac{2}{\pi \Gamma} \frac{C_{i}}{C_{i}^{\prime}} \int_{-\infty}^{+\infty} \times \\
& \times\left[1-\exp \left(-\frac{T C_{i}^{\prime} \Gamma^{2} / 4}{E^{2}+\Gamma^{2} / 4}\right)\right] \mathrm{d} E
\end{aligned}
$$

où $T=f a n \sigma_{0} t$ est l'épaisseur effective de la source.

Dans son article [2] qui traite de la surface des raies d'absorption, Blamey utilise une intégrale identique :

\begin{tabular}{|c|c|c|c|c|c|c|c|}
\hline & \multicolumn{2}{|c|}{ Absorbeur inox } & \multicolumn{2}{|c|}{ Absorbeur ferro cyanure } & Intensité & \multirow[b]{2}{*}{$\begin{array}{l}\text { Valeur } \\
\text { idéale }\end{array}$} \\
\hline & & $\begin{array}{l}\text { Intensité } \\
\text { relative \% }\end{array}$ & $\begin{array}{c}\Gamma \\
\mathrm{mm} / \mathrm{s}\end{array}$ & $\begin{array}{l}\text { Intensité } \\
\text { relative \% }\end{array}$ & $\begin{array}{c}\Gamma \\
\mathrm{mm} / \mathrm{s}\end{array}$ & $\begin{array}{c}\text { moyenne } \\
\%\end{array}$ & \\
\hline \multirow{4}{*}{$\mathrm{a}$} & - & - & - & - & - & - & - \\
\hline & $(1,6)$ & 39,09 & 0,46 & 39,67 & 0,32 & 39,4 & 37,5 \\
\hline & $(2,5)$ & 44,37 & 0,46 & 45,44 & 0,32 & 44,9 & 50 \\
\hline & $(3,4)$ & 16,54 & 0,44 & 14,90 & 0,28 & 15,7 & 12,5 \\
\hline \multirow{3}{*}{ b } & $(1,6)$ & 49,82 & 0,46 & 50,80 & 0,32 & 50,3 & 50 \\
\hline & $(2,5)$ & 31,12 & 0,42 & 30,42 & 0,28 & 30,8 & 33,3 \\
\hline & $(3,4)$ & 19,06 & 0,44 & 18,78 & 0,28 & 18,9 & 16,7 \\
\hline
\end{tabular}

$$
A=k \int_{-\infty}^{+\infty}\left[1-\exp \left(-n f \sigma_{0} \frac{\Gamma^{2} / 4}{(U-E)^{2}+\Gamma^{2} / 4}\right)\right] \mathrm{d} U
$$

Tableau I. - Résultats expérimentaux : a) avec champ appliqué ; b) en champ nul. Les valeurs idéales indiquées sont des valeurs théoriques qui supposent qu'il n'y a pas d'effet d'auto-absorption. 
à laquelle il donne la forme suivante :

$$
\begin{aligned}
A= & \frac{1}{2} \pi k \Gamma n f \sigma_{0} \exp \left(-\frac{1}{2} n f \sigma_{0}\right) \times \\
& \times\left[I_{0}\left(\frac{1}{2} n f \sigma_{0}\right)+I_{1}\left(\frac{1}{2} n f \sigma_{0}\right)\right]
\end{aligned}
$$

où $I_{0}$ et $I_{1}$ sont les fonctions de Bessel modifiées de première espèce et respectivement d'ordre 0 et 1 [4].

Avec le même procédé on réécrit l'expression (2) et l'on aboutit aux intensités relatives $Q_{i}$ :

$$
\begin{aligned}
Q_{i}= & \frac{P_{i}}{P_{0} N t}=C_{i} \exp \left(-C_{i}^{\prime} \frac{T}{2}\right) \times \\
& \times\left[I_{0}\left(\frac{C_{i}^{\prime} T}{2}\right)+I_{1}\left(\frac{C_{i}^{\prime} T}{2}\right)\right]
\end{aligned}
$$

les $Q_{i}$ sont normalisées quand l'épaisseur de la source est négligeable. Cette expression n'est pas très maniable, aussi calculons-nous le développement limité de $Q_{i}$ (utilisant les tables de [4])

$$
\begin{aligned}
Q_{i}= & C_{i}\left[1-C_{i}^{\prime} \frac{T}{4}+\left(C_{i}^{\prime} \frac{T}{4}\right)^{2}-\right. \\
& \left.-\frac{5}{6}\left(C_{i}^{\prime} \frac{T}{4}\right)^{3}+\theta\left(T^{4}\right)\right]
\end{aligned}
$$

qui nous conduit à l'expression approchée suivante :

$$
Q_{i}=\frac{C_{i}}{1+C_{i}^{\prime} \frac{T}{4}}
$$

L'essai de cette dernière expression avec des valeurs numériques montre qu'elle est correcte à $10^{-3}$ près (en valeur relative) pour $C_{i}^{\prime} T<2$.

En fait l'expérience ne nous donne pas directement les $Q_{i}$ (qui cessent d'être normalisés du fait de l'autoabsorption), mais les $Q_{i}^{\prime}$ normalisés, ainsi définis :

$Q_{i}^{\prime}=\frac{Q_{i}}{\sum Q_{i}}$

L'intensité perdue par auto-absorption est alors donnée, en valeur relative, par $1-\sum Q_{i}$.

Nous ne faisons pas ici, comme dans un article précédent [5], la discussion systématique des effets de polarisation. Nous la présenterons petit à petit, au fur et à mesure des besoins.

4. Source sous champ magnétique. Hypothèse de polarisation complète. - Lorsque l'aimantation du fer est perpendiculaire à la direction d'observation (expérience de la figure $2 a$ ), on réalise alors un cas idéal de polarisation rectiligne complète [1]. La polarisation parallèle d'une raie signifie que le champ électrique correspondant est parallèle au champ interne du noyau considéré (c'est-à-dire au champ magnétique appliqué).
Les intensités relatives des raies d'émission sont les suivantes [6] :

$$
\begin{aligned}
& C_{1}^{\|}=C_{6}^{\|}=3 / 8 ; \quad C_{2}^{\perp}=C_{5}^{\perp}=4 / 8 ; \\
& C_{3}^{\|}=C_{4}^{\|}=1 / 8
\end{aligned}
$$

(les coefficients $C_{1,3,4,6}$ correspondent à un état de polarisation parallèle, les $C_{2,5}$ à un état perpendiculaire). Comme, pour une raie donnée, les noyaux absorbeurs présentent le même état de polarisation que le noyau émetteur, ils pourront absorber le rayonnement; la probabilité d'absorption est alors égale à la probabilité d'émission. On peut donc écrire $C_{i}^{\prime}=C_{i}$, sans mentionner l'état de polarisation de la raie considérée, et utiliser la relation suivante :

$Q_{i}=\frac{C_{i}}{1+C_{i} \frac{T}{4}}$

$\operatorname{avec} \sum_{i=1}^{6} C_{i}^{\|}=1=\sum_{i=1}^{6} C_{i}^{\perp}$.

Nous avons tracé la variation calculée des $Q_{i}^{\prime}$ correspondants en fonction de $T$ sur la figure 3. La comparaison avec les valeurs expérimentales confirme qu'il $\mathrm{y}$ a bien des effets d'auto-absorption, mais suggère d'introduire d'autres corrections.

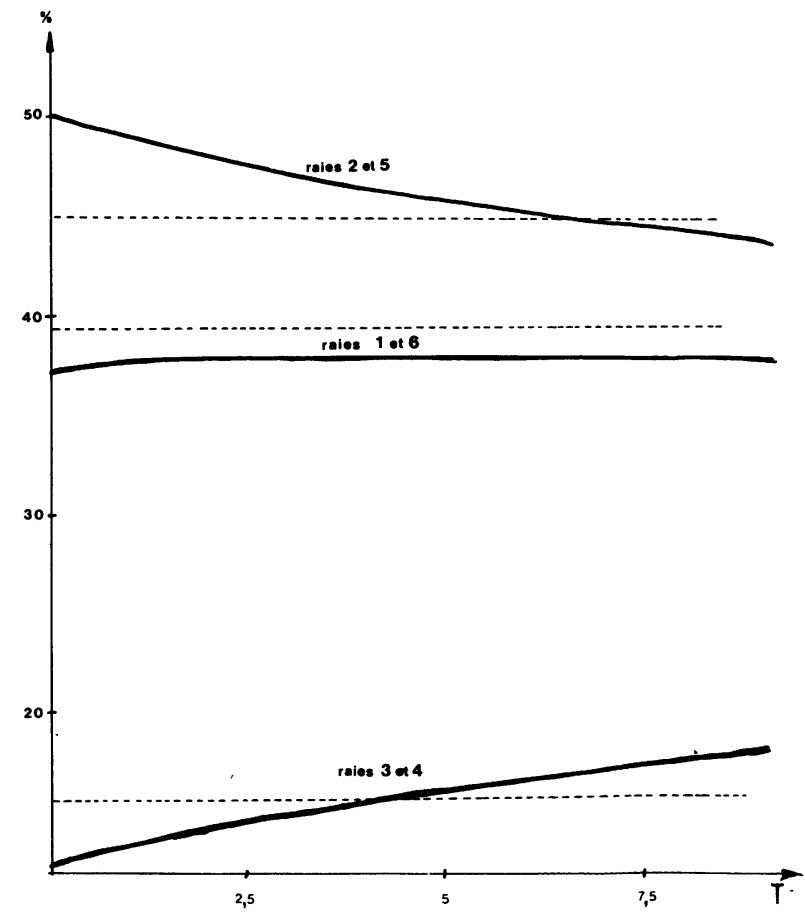

Fig. 3. - Intensités relatives calculées, pour une source polarisée linéairement à $100 \%$. En tirets : résultats des mesures.

[Relative intensities calculated for a completely linearly polarized source (in dashed line : results of measurements). ]

L'effet d'auto-absorption étant faible sur $Q_{1}^{\prime}$ (raies 1 et 6) on pourra alors utiliser ultérieurement cette raie comme référence. 
5. Source sous champ. Hypothèse de polarisation incomplète. - Compte tenu des différences entre les valeurs expérimentales et les valeurs calculées, supposons que l'état de polarisation de la source n'est pas complet. Considérons dans la source un noyau émetteur $N$ et un noyau absorbeur $N^{\prime}$.

5.1 Première hypothèse. - Supposons qu'en moyenne l'axe de quantification des noyaux émetteur et absorbeur est décalé d'un angle $\alpha$ (Fig. $4 a$ ) dans un plan $(\pi)$ perpendiculaire à la direction du rayonnement.
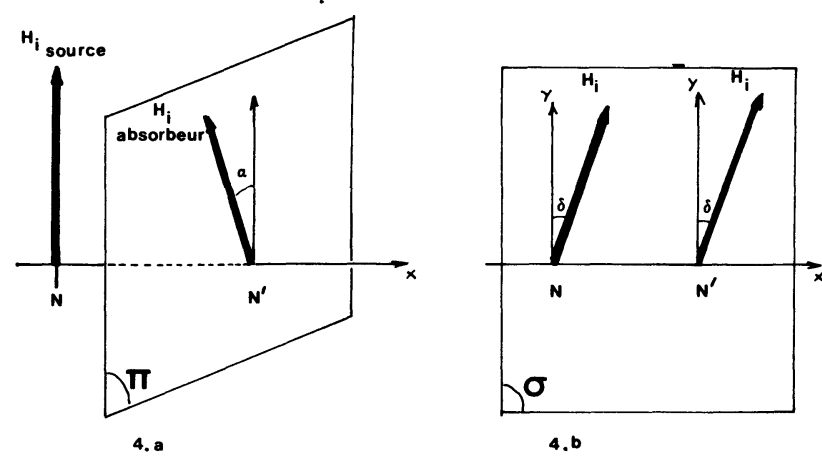

Fig. 4. - Hypothèses de polarisation incomplète (voir texte). [Incomplete polarisation assumptions (cf. text).]

L'équation (4) devient alors :

$Q_{i}=\frac{C_{i}}{1+C_{i} \cos ^{2} \alpha \frac{T}{4}}$.

En effet le $C_{i}$ du numérateur correspondant au noyau $N$ n'est pas modifié. Par contre la probabilité d'absorption de $N^{\prime}$ varie selon la loi de Malus, donc s'écrit $C_{i} \cos ^{2} \alpha$. (Dans ce cas les effets de réémission, que l'on a négligés, auraient produit de la biréfringence.)

Ceci revient à attribuer à la source une épaisseur effective réduite, $T \cos ^{2} \alpha$, qui ne permet pas de rendre compte de la valeur mesurée de $Q_{1}^{\prime}$ et donc nous conduit à rejeter cette première hypothèse.

5.2 DeUXIÈme HYPOTHÈSE. - Supposons que les noyaux émetteur et absorbeur ont bien des axes de quantification parallèles, mais que ces axes font un angle $(\pi / 2-\delta)$ avec la direction du rayonnement; tout se passe alors dans un plan $\sigma$ contenant la direction du rayonnement (Fig. 4b) (la raison de ce décalage étant la saturation magnétique incomplète de la source).

Les probabilités d'émission et d'absorption sont identiques entre elles, mais à la différence de ce qui a été dit auparavant (cf. 4), ici chaque raie contient les 2 états de polarisation rectiligne, // et $\perp$. L'équation (4) s'écrit alors :

$Q_{i}=Q_{i}^{\perp}+Q_{i}^{\|}=\frac{C_{i}^{\perp}}{1+C_{i}^{\perp} \frac{T}{4}}+\frac{C_{i}^{\|}}{1+C_{i}^{\|} \frac{T}{4}}$ avec $\sum_{i} C_{i}^{\|}=\sum_{i} C_{i}^{\perp}=1$

(Dans ce cas, les effets de réémission, négligés, auraient causé une rotation Faraday.)

Il convient alors de prendre pour les $C_{i}$ les valeurs suivantes [7], $O y$ restant la direction de référence pour les états de polarisation :

$$
\begin{array}{ll}
C_{1}^{\|}=C_{6}^{\|}=\frac{3}{8} & C_{1}^{\perp}=C_{6}^{\perp}=\frac{3}{8} \sin ^{2} \delta \\
C_{2}^{\|}=C_{5}^{\|}=0 & C_{2}^{\perp}=C_{5}^{\perp}=\frac{4}{8} \cos ^{2} \delta \\
C_{3}^{\|}=C_{4}^{\|}=\frac{1}{8} & C_{3}^{\perp}=C_{4}^{\perp}=\frac{1}{8} \sin ^{2} \delta
\end{array}
$$

Ces relations permettent de rendre compte des résultats expérimentaux en prenant

$$
\delta \sim 11 \quad \text { et } \quad T \sim 3,5
$$

la comparaison des valeurs calculées et expérimentales est faite sur le tableau II.

Tableau II. - Comparaison finale des intensités mesurées et calculées (exprimées en \%) à partir des données suivantes : a) $\delta=11,1^{\circ}$ et $T=3,7$ (source sous champ); b) $\alpha=0,10$ et $T=3$ (source en champ nul).

$\begin{array}{ccccc}\text { Raies } & \text { Exp (a) } & \text { Calc (a) } & \text { Exp (b) } & \text { Calc (b) } \\ - & - & - & - & - \\ 1,6 & 39,38 & 39,36 & 50,31 & 50,27 \\ 2,5 & 44,90 & 44,93 & 30,77 & 30,90 \\ 3,4 & 15,72 & 15,70 & 18,92 & 18,83\end{array}$

5.3 Remarques. - a) Compte tenu de la deuxième hypothèse on est conduit à prédire le taux de polarisation de la source :

- sur les raies 2 et 5 il reste égal à $100 \%$,

- sur les raies ( 1 et 6$)$ et ( 3 et 4 ) il devient :

$t=\frac{C_{1}^{\|}-C_{1}^{\perp}}{C_{1}^{\|}+C_{1}^{\perp}}=\frac{C_{3}^{\|}-C_{3}^{\perp}}{C_{3}^{\|}+C_{3}^{\perp}}=\frac{1-\sin ^{2} \delta}{1+\sin ^{2} \delta}$

ce qui conduit ici à : $t=93 \%$.

Globalement on aurait ainsi un taux de polarisation de l'ordre de $97 \%$ sur l'ensemble de raies de la source.

Dans la réalité il est probable que les 2 effets ( $1^{\mathrm{re}}$ et $2^{\mathrm{e}}$ hypothèses) sont présents simultanément, et l'on peut prévoir que la source aura un taux de polarisation moyen inférieur à la valeur calculée ici $(97 \%)$ [8].

b) Le calcul de $\sum_{i} Q_{i}$ avec les valeurs de $T$ et $\delta$ obtenues ci-dessus donne 0,74 , ce qui signifie que $26 \%$ environ de l'intensité résonnante émise par la source est perdue par auto-absorption. 


\section{Source en champ nul : hypothèse d'isotropie} parfaite. - En l'absence de champ magnétique appliqué, les atomes émetteurs et absorbeurs de la source sont répartis de manière quelconque par rapport à la direction du rayonnement et l'on suppose d'abord cette répartition isotrope.

Supposons de plus que les noyaux absorbeurs sont orientés au hasard par rapport aux noyaux émetteurs. (Ceci signifie en pratique que le rayonnement traverse des domaines magnétiques d'orientations diverses, ce qui n'est pas évident $a$ priori mais se trouve justifié par les résultats de cette étude.) Il n'est pas nécessaire de mentionner l'état de polarisation du noyau émetteur, et par suite l'équation (4) reste valable :

$Q_{i}=\frac{C_{i}}{1+C_{i} \frac{T}{4}},(4)$ avec $C_{i}^{\prime}=C_{i}$

et $\sum_{i=1}^{6} C_{i}=1$. Les $C_{i}$ ont pour valeur [6] :

$C_{1}=C_{6}=\frac{3}{6} ; \quad C_{2}=C_{5}=\frac{2}{6} ;$

$C_{3}=C_{4}=\frac{1}{6}$.

La figure 5 fait apparaître la variation calculée des $Q_{i}^{\prime}$ en fonction de $T$.

On constate que les courbes ne permettent pas de rendre compte des résultats expérimentaux. Il faut abandonner l'hypothèse d'une orientation isotrope.

7. Source en champ nul : hypothèse non isotrope. Par simplicité nous adoptons l'hypothèse suivante : une fraction $(1-\alpha)$ de la source possède effective-

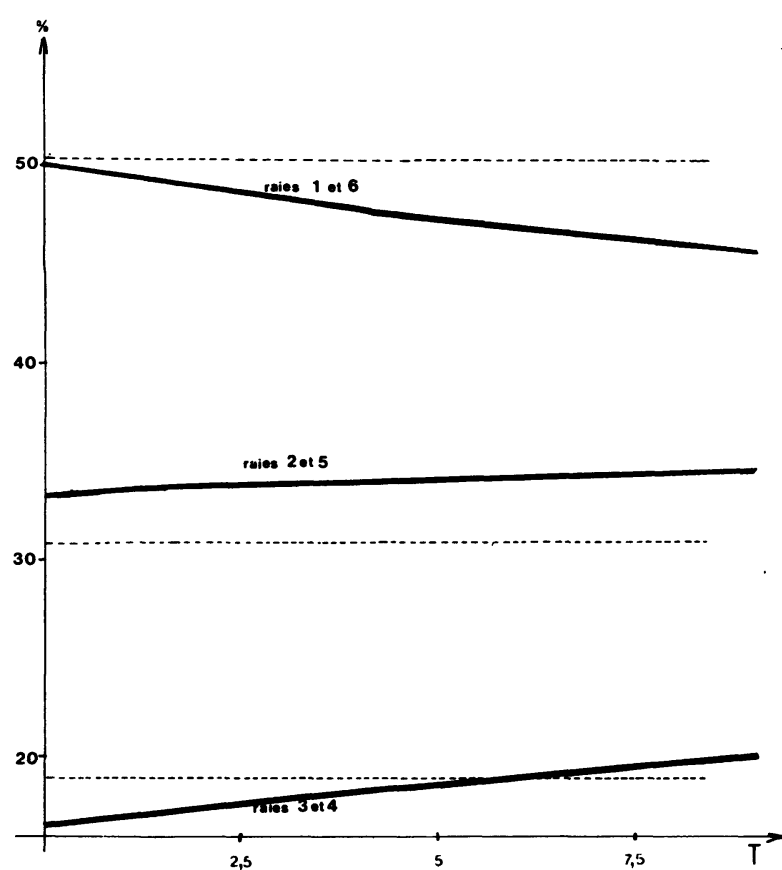

Fig. 5. - Intensités relatives calculées, pour une source isotrope. En tirets : résultat des mesures.

[Relative intensities calculated for an isotropic source (in dashed line : results of measurements).]

ment une répartition isotrope pour laquelle les relations (9) sont applicables. Le reste, c'est-à-dire la fraction $\alpha$, possède un champ hyperfin parallèle à la direction de propagation.

Cette hypothèse respecte la symétrie de révolution autour de la direction de propagation et chaque raie peut se trouver dans les deux états possibles de polarisation circulaire. Admettons comme précédemment que ces deux états sont également probables. Les coefficients d'émission et d'absorption sont égaux entre eux et sont donnés par [7] :

$$
\left.\begin{array}{l}
C_{1}^{+}=C_{1}^{-}=\frac{3}{12}(1-\alpha)+\frac{3}{8} \alpha \\
C_{2}^{+}=C_{2}^{-}=\frac{2}{12}(1-\alpha) \\
C_{3}^{+}=C_{3}^{-}=\frac{1}{12}(1-\alpha)+\frac{1}{8} \alpha
\end{array}\right\} \text { avec } \sum_{i=1}^{6} C_{i}^{+}=\sum_{i=1}^{6} C_{i}^{-}=1 .
$$

On utilise une équation semblable à l'équation (8) Ceci conduit finalement en comparant avec les résul$Q_{i}=Q_{i}^{+}+Q_{i}^{-}=\frac{C_{i}^{+}}{1+C_{i}^{+} \frac{T}{4}}+\frac{C_{i}^{-}}{1+C_{i}^{-} \frac{T}{4}}$. tats expérimentaux à :

$$
\alpha \sim 0,10 \text { et } \quad T \sim 3
$$


Les valeurs des intensités calculées sont reportées dans le tableau II. Le calcul montre aussi que $13 \%$ de l'intensité de la source est perdue par auto-absorption.

8. Discussion des résultats. - Les résultats de ces deux expériences semblent cohérents. Il faut tenir compte de l'imprécision des mesures d'intensité des raies $(\sim 0,5 \%$ ) qui introduisent sur $T$ une imprécision évaluée, d'après les courbes des figures 3 et 5 , à environ 1. Le résultat final est donc

$$
T=7 \pm 2
$$

On peut en déduire l'épaisseur de la couche de fer naturel traver'sée par le rayonnement; c'est :

$t=T /$ fan $_{0}=(11 \pm 3) \mu \mathrm{m}$.

Cette valeur s'explique mal par la profondeur de diffusion du ${ }^{57} \mathrm{Co}$ annoncée par le constructeur : $6 \mu \mathrm{m}$. Il est possible que la forme de la surface soit assez irrégulière pour que la couche de fer se présente souvent de manière oblique au rayonnement. Il est possible aussi que l'estimation du constructeur soit peu précise et que $\rho(x)$ ne soit pas constant.
La signification des corrections introduites doit être cherchée dans l'état physique de la source. On peut supposer la source formée de petits grains, dont chacun présente a priori, s'il n'a pas la forme sphérique, une aimantation facile dans sa plus grande dimension, pour raison de champ démagnétisant. Ce que l'on observe à champ nul proviendrait alors d'une texture où les grains seraient plus volontiers allongés dans la direction du rayonnement. Les résultats sous champ indiqueraient que 0,4 tesla ne suffit pas à surmonter complètement le champ démagnétisant.

Pour obtenir une source polarisée à $100 \%$, il vaudrait mieux que la matrice soit sous la forme d'une feuille mince de fer; mais alors elle serait loin d'être isotrope en champ nul.

9. Conclusion. - Les effets d'auto-absorption dans une source commerciale de ${ }^{57} \mathrm{Co}$ dans du fer métallique ne sont pas négligeables. Ils sont même mesurables et l'on a pu en déduire l'épaisseur effective de la couche traversée, qui semble nettement supérieure à ce qui correspond aux données du constructeur.

De plus, il paraît irréaliste qu'une seule source prétende aux deux qualités suivantes : isotropie en champ nul, polarisation à $100 \%$ en présence d'un champ magnétique modéré.

\section{Bibliographie}

[1] Imbert, P., Thèse CEA, Saclay 1965, J. Physique 27 (1966) 429.

[2] Blamey, P. J., Nucl. Instrum. Methods 142 (1977) 553-557.

[3] Margulies, S. and Ehrman, J. R., Nucl. Instrum. Methods 12 (1961) 131

[4] Handbook of mathematical tables (Chemical Rubber Co).

[5] HeNRY, M., VARREt, F., en cours de publication à Nucl. Instrum. Methods.

[6] JANOT, C., L'effet Mössbauer et ses applications (Masson Editeur) 1972 (page 42 et page 39).
Frauenfelder, H., Nagle, G. E., Taylor, R. D., CochRAN, D. R. F., VisSCher, W. M., Phys. Rev. 126 (1962) 1065.

[7] VARret, F., Communication privée.

[8] Nos premières mesures donnent $t \gtrsim 90 \%$ (absorbeur fer aimanté).

[9] Housley, R. M., Gonser, U., Phys. Rev. 171 (1968) 480. Blume, M., Kistner, O. C., Phys. Rev. 171 (1968) 417. 